\title{
Epidemiological analysis of group A streptococci recovered from patients in China
}

\author{
Hong-bo Jing, ${ }_{1}^{1}$ Boa-an Ning, ${ }^{1}$ Huai-jie Hao, ${ }^{1}$ Yu-ling Zheng, ${ }_{1}^{1}$ \\ Dong Chang, ${ }^{2}$ Wei Jiang ${ }^{2}$ and Yong-qiang Jiang ${ }^{1}$
}

Correspondence

Yong-qiang Jiang

jiangyq@nic.bmi.ac.cn

Received 13 July 2005

Accepted 15 March 2006

\author{
${ }^{1}$ State Key Laboratory of Pathogen and Biosecurity, The Institute of Microbiology and \\ Epidemiology, Academy of Military Medical Sciences, no. 20 Dongda Street, Fengtai District, \\ Beijing, 100071, People's Republic of China \\ 2304 Hospital, Haidian District, Beijing, 100037, People's Republic of China
}

\begin{abstract}
Since the mid-1980s, there has been a resurgence of severe forms of invasive group $A$ streptococcal (GAS) disease in many countries and regions. However, there has not been any systemic epidemiologic analysis of GAS disease reported in mainland China. To analyse the molecular epidemiology of GAS disease, 86 strains from patients in different regions of mainland China were collected. The collection sites included blood, pus, wounds, the epipharynx and other sites. A total of 21 different emm types were identified in the isolates. In both invasive and non-invasive isolates, M1 (29.1\%) and M12 (23.3\%) were the most prevalent types, a different distribution to $\mathrm{M}$ type distributions reported in other countries. Furthermore, minor emm gene sequence alterations were noted for six types. Several important GAS virulence factors were detected by PCR using specific primers. The spe $B$ and slo genes were detected in all isolates and were species specific. Four superantigen genes, speA, speC, smeZ and ssa, were found in $52 \%$ (45/86), $51 \%(44 / 86), 82 \%(71 / 86)$ and $23 \%(27 / 86)$ of isolates, respectively. M1 isolates harboured more speA (84\%) and fewer speC genes (44\%), while M12 isolates had fewer speA (35\%) and more speC genes (100\%). There was also an association between some virulence genes and isolation sites, perhaps due to the correlation between the emm type distribution and virulence gene occurrence. For two important virulence genes related to necrotizing fasciitis, the sil gene was only carried by 11 of 86 isolates, and no sil gene contained the start codon ATA. The sla gene rarely occurred in GAS isolates, only four of 86 GAS strains being positive, including two isolates obtained from blood. In antimicrobial susceptibility tests, the overall rate of drug resistance in GAS isolates was higher than reported rates in other countries, and the resistance rates to erythromycin, tetracycline and clindamycin were $91 \cdot 8,93.4$ and $80 \%$, respectively. This epidemiological study may help to understand the pathogenesis of GAS disease and aid in vaccine development.
\end{abstract}

\section{INTRODUCTION}

Group A streptococci are an important group of Grampositive extracellular bacterial pathogens that cause a variety of diseases, including pharyngitis, scarlet fever, cellulitis, bacteraemia, impetigo, acute rheumatic fever, glomerulonephritis, necrotizing fasciitis (NF) and streptococcal toxic shock syndrome (STSS). Since the mid-1980s, an increase in the incidence of invasive infections caused by group A streptococci has been reported in many countries and regions (Banks et al., 2002; Carapetis et al., 1995; Holm et al., 1992; Kaul et al., 1997; Kiska et al., 1997; O’Brien et al., 2002; Chiou et al., 2004; Ho et al., 2003). This dramatic rise may

Abbreviations: GAS, group A streptococcal; NF, necrotizing fasciitis; STSS, streptococcal toxic shock syndrome. be due to changes in specific virulence factors (Vlaminckx et al., 2003; Chatellier et al., 2000), hence strain characterization has assumed great importance.

Among the many virulence factors produced by group A streptococci, the M protein encoded by the emm gene is considered to be a major factor. About 150 different $\mathrm{M}$ protein gene sequence types have been documented (Bisno et al., 2003; Cunningham, 2000). It has been reported that strains of certain $\mathrm{M}$ types are epidemiologically associated with particular clinical syndromes. For example, the majority of recently reported invasive group A streptococcal (GAS) infections have been caused by strains of the M1 or M3 type, and infections with these organisms are associated with increased mortality (O'Brien et al., 2002; Chatellier et al., 2000; Musser et al., 1995; Ikebe \& Wader, 2002). Moreover, a 
particularly virulent subclone of M1 associated with invasive infections has been spreading globally for more than 20 years. However, the mechanism(s) by which any $\mathrm{M}$ type causes a specific clinical manifestation still remains controversial.

Among the virulence factors that regulate GAS pathogenicity, the known extracellular products, including streptolysin O (SLO), streptococcal pyrogenic exotoxin B (SpeB) and superantigen toxins, are principal contributors. The virulence-related superantigens are the pyrogenic exotoxins SpecA, SpeC, SSA and SMEZ. They are mitogenic for certain $\mathrm{T}$-cell subsets and do not require processing by antigenpresenting cells, and thus can activate a much larger number of $\mathrm{T}$ cells than conventional antigens and release excessive amounts of inflammatory cytokines (Bisno et al., 2003; Cunningham, 2000; Kotb, 1995). They are encoded on various phage genomes and are associated with gene variation. SLA is a prophage-encoded secreted extracellular phospholipase $\mathrm{A}_{2}$ in GAS strains and is associated with host-pathogen interaction (Banks et al., 2003; Beres et al., 2002; Nagiec et al., 2004). The sil gene is the streptococcal invasion locus, which controls GAS spread into deeper tissues and may be involved in DNA transfer (Hidalgo-Grass et al., 2002, 2004).

Studies of GAS distribution have been performed worldwide, but there are few data from mainland China, where the epidemiology of GAS may be different. In Asia, studies from Malaysia, Thailand and Hong Kong have revealed a different GAS type distribution (Chiou et al., 2004; Ho et al., 2003). In mainland China, a similar GAS infection increase has also been observed in recent years. Severe infections, such as NF and STSS, caused by group A streptococci were earlier almost unknown. To obtain a better understanding of the epidemiology of GAS infections in mainland China, we performed a molecular epidemiology analysis on GAS isolates obtained from patients in China, including GAS emm typing, detection of some virulence factors, and antibioticsusceptibility tests.

\section{METHODS}

Bacterial strains. A total of 86 strains of group A streptococci were recovered from patients in different regions of mainland China from 2000 to 2004 . The sources of the specimens included hospitalized patients, as well as outpatients from emergency rooms and specific outpatient clinics. All GAS strains were grown on blood agar plates in $5 \% \mathrm{CO}_{2}$ and at $37^{\circ} \mathrm{C}$ overnight. All isolates were $\beta$-haemolytic and their identity was confirmed using API strips. Invasive isolates were exclusively from blood $(n=10)$. Non-invasive isolates were obtained from the epipharynx $(n=47)$, pus $(n=7)$, wounds $(n=7)$ and other sources $(n=15)$.

DNA preparation. The DNA preparation of GAS stains was performed as described by the USA Centers for Disease Control and Prevention (CDC) (http://www.cdc.gov/ncidod/biotech/strep/protocols.htm). Briefly, one loopful of bacteria was suspended in $300 \mu \mathrm{l}$ $0 \cdot 85 \% \mathrm{NaCl}$ and heated for $15 \mathrm{~min}$ at $70^{\circ} \mathrm{C}$. The samples were centrifuged and resuspended in $50 \mu \mathrm{l}$ TE buffer $(10 \mathrm{mM}$ Tris, $1 \mathrm{mM}$ EDTA, pH 8) supplemented with $10 \mu \mathrm{l}$ mutanolysin $\left(3000 \mathrm{U} \mathrm{ml}^{-1}\right.$ ) and $2 \mu \mathrm{l}$ hyaluronidase $\left(30 \mathrm{mg} \mathrm{ml}^{-1}\right)$. The mixture was then incubated for $30 \mathrm{~min}$ at $37^{\circ} \mathrm{C}$, heated at $100^{\circ} \mathrm{C}$ for $10 \mathrm{~min}$ and briefly clarified by centrifugation. The supernatant including genomic DNA was used as the template for PCR.

emm genotyping. The $M$ genotypes of strains were identified as described at the CDC website (http://www.cdc.gov/ncidod/biotech/ strep/strepindex.html). Briefly, the $\mathrm{emm}$ gene encoding the $\mathrm{M}$ protein was amplified with the genomic DNA described above as template and P1 [5'-tatt(c/g)gcttagaaaattaa- $\left.3^{\prime}\right]$ and P2 (5'-gcaagttcttcagcttgttt$3^{\prime}$ ) as primers. The cycling parameters were $94^{\circ} \mathrm{C}$ for $15 \mathrm{~s}, 46^{\circ} \mathrm{C}$ for $30 \mathrm{~s}$, and $72^{\circ} \mathrm{C}$ for $1 \mathrm{~min} 15 \mathrm{~s}$ for the first 10 cycles, and then $94^{\circ} \mathrm{C}$ for $15 \mathrm{~s}, 46^{\circ} \mathrm{C}$ for $30 \mathrm{~s}, 72^{\circ} \mathrm{C}$ for $1 \mathrm{~min} 15 \mathrm{~s}$ (with a $10 \mathrm{~s}$ increment for each of the subsequent 19 cycles) for the subsequent 20 cycles. The PCR product was sequenced with primer emmseq2 (5'-tattcgcttagaaaattaaaaacagg- $\left.3^{\prime}\right)$ and the first $160 \mathrm{bp}$ of the $5^{\prime} \mathrm{emm}$ sequence of every strain was compared with the sequences in the CDC emm database (http://www.cdc.gov/ncidod/biotech/strep/strepblast.htm) to determine $\mathrm{emm}$ types. This sequence includes 70 bases encoding the signal peptide and 90 bases encoding the first 30 amino acids of the mature protein. The subtypes were assigned to exact $150 \mathrm{bp}$ sequences encoding the $\mathrm{N}$-terminal 50 residues of the mature $\mathrm{M}$ protein.

Detection of virulence genes. Virulence genes, including $\operatorname{sep} A$, sepB, sepC, slo, ssa, smeZ, sla and sil, were detected by PCR with primers as shown in Table 1. Amplification of all genes was performed with an initial $5 \mathrm{~min}$ denaturation step at $94^{\circ} \mathrm{C}$ followed by 30 cycles of denaturation at $94{ }^{\circ} \mathrm{C}$ for $30 \mathrm{~s}, 30 \mathrm{~s}$ of annealing at the appropriate temperature for each gene, as specified in Table 1 , and $60 \mathrm{~s}$ of extension at $72{ }^{\circ} \mathrm{C}$ with a final extension step at $72{ }^{\circ} \mathrm{C}$ for $7 \mathrm{~min}$.

Susceptibility tests. Susceptibility testing to erythromycin, clindamycin, tetracycline, ceftriaxone, penicillin G, vancomycin, ciprofloxacin and chloramphenicol was performed on some isolates. MICs were determined by the broth microdilution method. All results were interpreted according to National Commmittee for Clinical Laboratory Standards criteria with a final inoculum of $10^{5}$ c.f.u. $\mathrm{ml}^{-1}$. Streptococcus pneumoniae ATCC 49619 was used as a control strain. The data were analysed with WHONET 5 software.

Statistical analysis. The chi square test was used for statistical analysis with a $P$ value $<0 \cdot 05$ considered significant. The SPSS 10.0 statistical package was used for all analyses.

\section{RESULTS}

\section{Distribution of emm types}

A total of $21 \mathrm{M}$ types were identified among the 86 clinical isolates. In descending order of frequency, the prevalent $\mathrm{M}$ types (with a frequency $>3 \%$ in the population) in mainland China were M1 $(29 \cdot 1 \%)$, M12 $(23 \cdot 3 \%)$, M8 $(7 \cdot 0 \%), \operatorname{M} 18(5 \cdot 8 \%), \operatorname{M} 80(4 \cdot 7 \%)$ and M28 (4.7\%). The other M types (M4, M66, M77, M94, M101, M3, M6, M23, M44, M63, M64, M75, M85, M86 and M88) were detected in only one or two isolates. M1 and M12 together accounted for 60.0 and $51.3 \%$ of the invasive and non-invasive isolates, respectively.

A total of six different $\mathrm{M}$ types, including M1, M12, M18, M80, M6 and M86, were found in the 10 invasive isolates. A total of 19 different $M$ types were identified in the 76 noninvasive isolates. Besides the most prevalent M1 and M12 
Table 1. Primers used for PCR of virulence genes

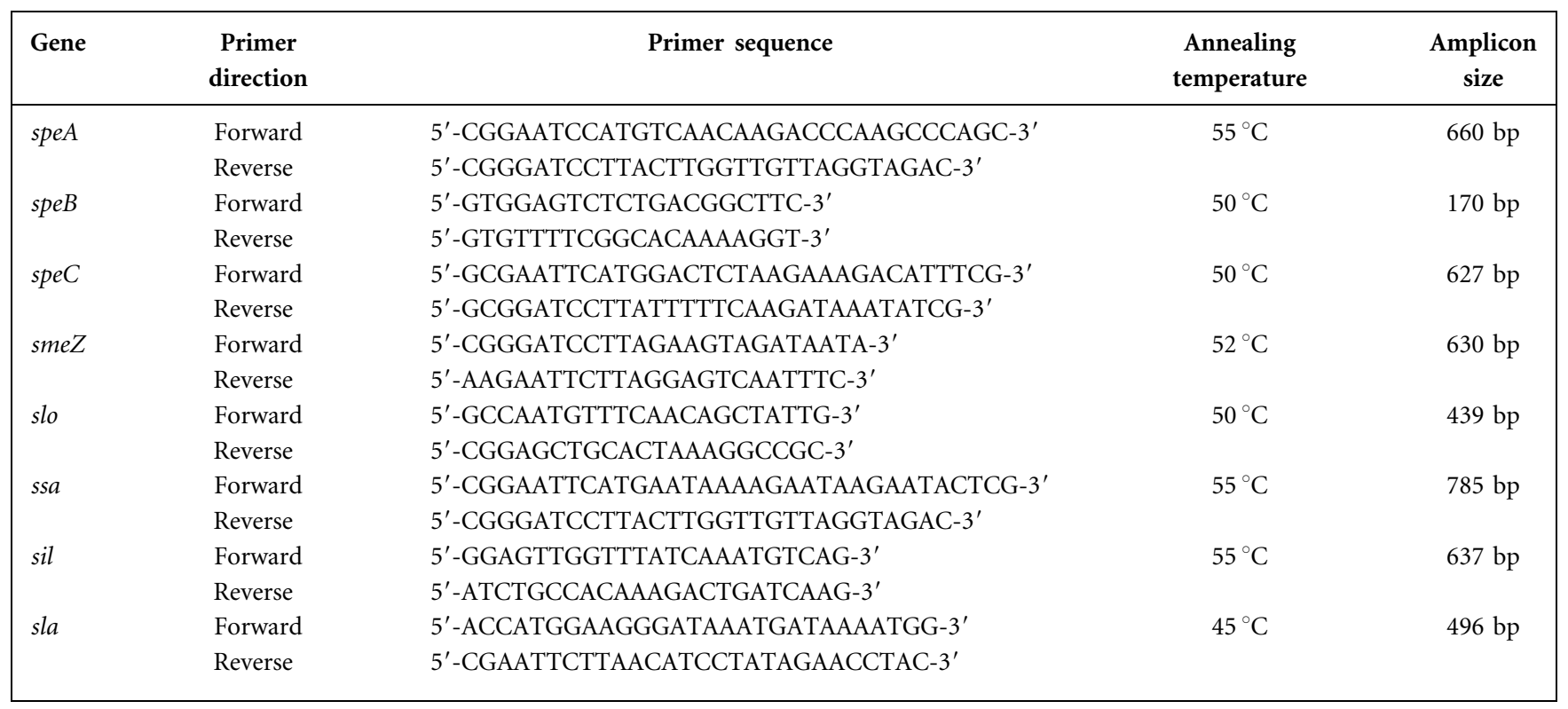

types, other common types in non-invasive isolates (with a frequency $>3 \%$ ) were M8 (7.9\%), M18 (5.3\%), M28 $(5 \cdot 3 \%)$, M80 (3.9\%) and M101 (3.9\%). M8 and M28 were absent in the 10 invasive isolates and more frequent in the non-invasive isolates $(P<0 \cdot 05)$.

Only few emm sequence variations were found in our study. In nine of 86 isolates, types emm1.22, emm12.13, emm3.1, emm6.10, emm18.12 and emm86.1 were found to contain one to six base substitutions relative to $M$ type reference strains, while no deletions and insertions were found in other isolates. Most of these subtype variants occurred in one or two isolates (Table 2). The remaining 77 isolates all had DNA sequences identical to the corresponding reference emm sequences.

Table 2. Allelic variation(s) in different emm subtypes

\begin{tabular}{|c|c|c|}
\hline emm subtype & $\begin{array}{l}\text { Number } \\
\text { of isolates }\end{array}$ & $\begin{array}{l}\text { Allelic variation(s) } \\
\quad \text { in } \mathrm{emm}^{*}\end{array}$ \\
\hline emm 1.22 & 2 & $55 \mathrm{C}$ to $\mathrm{T}$ \\
\hline emm 12.13 & 1 & $119 \mathrm{G}$ to $\mathrm{A}$ \\
\hline emm3.1 & 2 & $92 \mathrm{~A}$ to $\mathrm{C}, 122 \mathrm{G}$ to $\mathrm{A}$ \\
\hline emm 18.12 & 2 & $\begin{array}{l}6 \mathrm{~T} \text { to } \mathrm{C}, 25 \mathrm{G} \text { to } \mathrm{A}, 89 \mathrm{~T} \\
\text { to } \mathrm{A}, 114 \mathrm{~T} \text { to } \mathrm{A}\end{array}$ \\
\hline emm86.1 & 1 & $\begin{array}{l}51 \mathrm{C} \text { to } \mathrm{A}, 71 \mathrm{C} \text { to } \mathrm{G}, 113 \mathrm{~T} \\
\text { to } \mathrm{G}, 118 \mathrm{G} \text { to } A, 127 \mathrm{~A} \\
\text { to } \mathrm{G}, 133 \mathrm{G} \text { to } A\end{array}$ \\
\hline emm6.10 & 1 & 38 A to $\mathrm{G}$ \\
\hline
\end{tabular}

${ }^{\star}$ Numbered from the base encoding the $\mathrm{N}$ terminus of the mature 50 amino acid protein.

\section{Identification of virulence genes}

The virulence genes speA, speB, speC, slo, ssa, smeZ, sla and sil were amplified by PCR. As shown in Table 3, speB and slo were detected in all GAS isolates, proving to be species specific. Four superantigen genes, sepA, spec, smeZ and $s s a$, were found in $52 \%(45 / 86), 51 \%(44 / 86), 82 \%$ (71/86) and $23 \%(27 / 86)$ of GAS isolates, respectively. The genes sla and sil rarely occurred in our isolates and were identified in $4 \cdot 7 \%(4 / 86)$ and $12 \cdot 8 \%(11 / 86)$ of isolates, respectively.

The relationship between the distribution of emm types and virulence genes is also shown in Table 3. For instance, M1 isolates harboured more speA (84\%) and fewer speC genes (44\%), while M12 isolates carried fewer speA (35\%) and more speC genes $(100 \%)$. The speA gene was prevalent in the M80 (100\%), M1 (84\%) and M18 (60\%) isolates, while the speC gene was prevalent in the M12 (100\%), M118 (60\%) and M1 (44\%) isolates, respectively. Analysis of all strains revealed that $24 \%$ of the isolates carried both speA and speC. Interestingly, three important superantigen genes, speA, speC and ssa, were absent in all six non-invasive M8 isolates. The emm types of four isolates containing the sla gene were M18 (2/5), M101 (1/3) and M86 (1/1). The emm types of 11 isolates containing the sil gene were M1 (1/24), M18 (2/5), M4 (2/2), M94 (2/2), M101 (3/3) and M86 (1/1), but no sil gene contained the start codon ATA. Interestingly, two M4 strains contained one nucleotide substitution $(\mathrm{C} \rightarrow \mathrm{T})$ at -20 , while the sil sequences of other strains were same.

The association of virulence genes and source is shown in Table 4. The speA gene was found in $70 \%$ of isolates from blood and $51 \%$ of isolates from the epipharynx, while speC was found in $40 \%$ of isolates from blood and $62 \%$ of 
Table 3. Distribution of virulence genes among $M$ types

\begin{tabular}{|c|c|c|c|c|c|c|c|c|c|}
\hline \multirow[t]{2}{*}{ M type } & \multirow{2}{*}{$\begin{array}{l}\text { Number } \\
\text { of strains }\end{array}$} & \multicolumn{8}{|c|}{ Number of isolates carrying virulence gene: } \\
\hline & & speA & speB & speC & smeZ & $s s a$ & slo & sil & sla \\
\hline M1 & 25 & 21 & 25 & 11 & 19 & 10 & 25 & 1 & 0 \\
\hline M12 & 20 & 7 & 20 & 20 & 17 & 8 & 20 & 0 & 0 \\
\hline M8 & 6 & 0 & 6 & 0 & 6 & 0 & 6 & 0 & 0 \\
\hline M18 & 5 & 3 & 5 & 3 & 5 & 1 & 5 & 2 & 2 \\
\hline M80 & 4 & 4 & 4 & 1 & 4 & 0 & 4 & 0 & 0 \\
\hline M28 & 4 & 1 & 4 & 0 & 3 & 1 & 4 & 0 & 0 \\
\hline M101 & 3 & 1 & 3 & 0 & 3 & 0 & 3 & 3 & 1 \\
\hline M4 & 2 & 0 & 2 & 2 & 2 & 2 & 2 & 2 & 0 \\
\hline M66 & 2 & 0 & 2 & 0 & 2 & 0 & 2 & 0 & 0 \\
\hline M77 & 2 & 1 & 2 & 2 & 2 & 0 & 2 & 0 & 0 \\
\hline M94 & 2 & 1 & 2 & 1 & 2 & 1 & 2 & 2 & 0 \\
\hline M3 & 2 & 2 & 2 & 2 & 0 & 2 & 2 & 0 & 0 \\
\hline M6 & 1 & 1 & 1 & 1 & 0 & 1 & 1 & 0 & 0 \\
\hline M23 & 1 & 0 & 1 & 0 & 1 & 1 & 1 & 0 & 0 \\
\hline M44 & 1 & 1 & 1 & 0 & 0 & 0 & 1 & 0 & 0 \\
\hline M63 & 1 & 1 & 1 & 0 & 1 & 0 & 1 & 0 & 0 \\
\hline M64 & 1 & 0 & 1 & 0 & 1 & 0 & 1 & 0 & 0 \\
\hline M75 & 1 & 1 & 1 & 0 & 1 & 0 & 1 & 0 & 0 \\
\hline M85 & 1 & 0 & 1 & 1 & 0 & 0 & 1 & 0 & 0 \\
\hline M86 & 1 & 0 & 1 & 0 & 1 & 0 & 1 & 1 & 1 \\
\hline M88 & 1 & 0 & 1 & 0 & 1 & 0 & 1 & 0 & 0 \\
\hline Total & 86 & 45 & 86 & 44 & 71 & 27 & 86 & 11 & 4 \\
\hline
\end{tabular}

isolates from the epipharynx. The speC gene was significantly over-represented among isolates from the epipharynx. The occurrence rates of speA, speC and ssa in wound isolates were much lower than in isolates from other sources. This could be explained by the reduced need for GAS virulence factors to establish infection after the destruction of the skin barrier. Because two out of four strains containing the sla gene were obtained from blood, the sla gene was more frequent in invasive isolates than non-invasive isolates overall $(2 / 10$ versus $2 / 64 ; P<0 \cdot 01)$. Consistent with our expectation, none of six M8 isolates lacking speA, speC and ssa caused invasive infection in blood.

\section{Susceptibility testing}

We performed antimicrobial susceptibility testing with eight common antibiotics. MICs are presented in Table 5 for 61 GAS isolates. We found resistance to erythromycin, tetracycline and clindamycin among all GAS isolates. Overall, $80,91 \cdot 8$ and $93.4 \%$ of isolates were resistant to clindamycin, erythromycin and tetracycline, respectively. All 61 strains tested were susceptible to ceftriaxone, vancomycin and chloramphenicol, and 85.2 and $98.4 \%$ of these isolates were susceptible to ciprofloxacin and penicillin $\mathrm{G}$, respectively.

Table 4. Association of virulence genes and strain source

\begin{tabular}{|c|c|c|c|c|c|c|c|c|c|}
\hline Source & $\begin{array}{l}\text { Number } \\
\text { of cases }\end{array}$ & \multicolumn{8}{|c|}{ Number (\%) of isolates with gene: } \\
\hline Wound & 7 & $1(14 \%)$ & $7(100 \%)$ & $2(29 \%)$ & $7(100 \%)$ & $1(14 \%)$ & 0 & 0 & $6(86 \%)$ \\
\hline Epipharynx ${ }^{*}$ & 47 & $24(51 \%)$ & $47(100 \%)$ & $29(62 \%)$ & $47(100 \%)$ & $19(40 \%)$ & $1(2 \cdot 1 \%)$ & $7(15 \%)$ & $36(77 \%)$ \\
\hline Pus & 7 & $4(57 \%)$ & $7(100 \%)$ & $2(29 \%)$ & $7(100 \%)$ & $1(14 \%)$ & $1(14 \%)$ & $2(29 \%)$ & $7(100 \%)$ \\
\hline
\end{tabular}

${ }^{\star}$ Epipharynx includes throat, nose and sputum.

†Other includes vaginal secretions, urine and unknown. 
Table 5. Susceptibility of 61 GAS isolates to various antibiotics

\begin{tabular}{|c|c|c|c|c|c|c|}
\hline \multirow[t]{2}{*}{ Antibiotic } & \multicolumn{3}{|c|}{ Antibiotic MIC ( $\left.\mathrm{mg} \mathrm{l}^{-1}\right)$} & \multicolumn{3}{|c|}{ Susceptibility (\%) } \\
\hline & Range & $\mathrm{MIC}_{50}$ & $\mathrm{MIC}_{90}$ & Resistant & Intermediate & Susceptible \\
\hline Clindamycin & $0 \cdot 06-4$ & 4 & 4 & 80 & $1 \cdot 7$ & $18 \cdot 3$ \\
\hline Ciprofloxacin & $0 \cdot 06-2$ & $0 \cdot 5$ & 2 & 0 & $14 \cdot 8$ & $85 \cdot 2$ \\
\hline Erythromycin & $0 \cdot 03-4$ & 4 & 4 & $91 \cdot 8$ & $1 \cdot 6$ & $6 \cdot 6$ \\
\hline Tetracycline & $0 \cdot 12-32$ & 32 & 3 & $93 \cdot 4$ & 0 & $6 \cdot 6$ \\
\hline Vancomycin & $0 \cdot 25-0 \cdot 5$ & $0 \cdot 25$ & $0 \cdot 25$ & 0 & 0 & 100 \\
\hline Chloramphenicol & $2-4$ & 4 & 4 & 0 & 0 & 100 \\
\hline
\end{tabular}

\section{DISCUSSION}

This is the first molecular epidemiological study of GAS isolates causing invasive and non-invasive infection in different regions of mainland China. Our study revealed unique $\mathrm{M}$ type profiles in mainland China which were different to $\mathrm{M}$ type distributions reported in Hong Kong. The prevalent $M$ types in mainland China were M1 $(29 \cdot 1 \%)$, M12 (23.3\%), M8 (7·0\%), M18 (5.8\%), M80 $(4 \cdot 7 \%)$ and M28 (4.7\%), compared to the prevalent M types in Hong Kong, M12 (20.6\%), M1 (15.0\%), M58 $(10 \cdot 3 \%)$, M $4(9 \cdot 3 \%)$, M53 (4.7\%) and M49 (4.7\%) (Ho et al., 2003). M1 was the most prevalent type among isolates from invasive disease as well as in non-invasive isolates. M1 was equally prevalent in both invasive and non-invasive isolates in previous epidemiological studies performed in North Carolina, USA, and Hong Kong (Kiska et al., 1997; Ho et al., 2003). The M3 type has been reported to be prevalent in invasive infections such as NF and STSS in the USA, Canada and other countries (Banks et al., 2002; Carapetis et al., 1995; Kaul et al., 1997; O’Brien et al., 2002; Ikebe \& Wada, 2002; Banks et al., 2003), but only two M3 type GAS strains were found in the present study, and they were both non-invasive. Hidalgo-Grass et al. $(2002,2004)$ have reported that the M14 type is associated with invasive infections in Israel, but this M type was not observed at all in our study, nor in Hong Kong or Taiwan (Chiou et al., 2004; Ho et al., 2003). This suggests that the GAS M type distribution in China differs from that in other countries. The lack of M3 and M14 strains may account for the fact there were fewer clinically severe infections such as NF and STSS caused by group A streptococci in mainland China.

Only minor emm gene sequence alterations were noted for six emm types in our study. Allelic variation in the $\mathrm{emm}$ genes of our isolates, compared to reference sequences, was predominantly due to single-base substitutions. This study also showed that the GAS emm type distribution in mainland China differs from those in Thailand and Hong Kong (Chiou et al., 2004; Ho et al., 2003).

In order to study the factors contributing to the aggressive clinical picture seen with some GAS infections, the isolates were tested for the presence of different toxin genes. The streptococcal pyrogenic exotoxin B (SepB) is a highly conserved extracellular cysteine protease that is associated with pyrogenicity, T-lymphocyte mitogenicity, and the ability to increase susceptibility to endotoxic shock in individuals infected with group A streptococci (Collin \& Olsen, 2001; Watanabe et al., 2002). Louie \& Simor (1988) reported that $s p e B$ was positive in all GAS isolates by PCR assay, but the assay was negative in all patients without culture or serological evidence of streptococcal infection. Our results were the same, indicating that PCR detection of the speB gene in GAS strains may be a useful method to confirm GAS infection.

The isolates were tested for the presence of the phageencoded superantigens. It has been reported that speA is located in phages T12, 270 and 49 (Yu \& Ferretti, 1991) and speC in phage CS112 (Goshorn \& Schlievert, 1989). We showed that these toxin genes were exclusively associated with particular M types. Some of these observed associations between $\mathrm{M}$ types and toxin genes have been reported by others. For instance, Schmitz et al. (2003) have found that all but one of $239 \mathrm{M} 1$ and M3 strains carry a speA allele and lack a speC gene, suggesting that the presence of speA affects the acquisition of a speC gene in M1 and M3 types. Vlaminckx et al. (2003) speculated that the exclusive combination of speA and smeZ in M1, which encode proteins known to be immunoactive, might contribute to intrinsic virulence. This specific set of virulence genes might make them more efficient as pathogens, findings in accordance with our study.

The presence of speA, as opposed to speC, was positively correlated with invasive infection in blood. This finding was consistent with the notion that speA is an important virulence factor required to cause invasive infection. The speC gene occurred more frequently in strains obtained from the epipharynx than in those obtained from other sources, a finding supportive of the conclusion that speC and bacteriophage induction are pharyngeal-cell dependent. Broudy et al. (2001) have reported that speC is induced along with phage CS112 when group A streptococci are co-cultured with human pharyngeal cells.

It has been reported that the prophage-encoding sla is not present in M3 GAS strains recovered before 1987, and that 
strains containing an sla gene are more likely to come from a severe infection such as NF. When the resurgence of severe invasive disease occurred in North America, Europe and Japan (Banks et al., 2003; O'Brien et al., 2002), Nagiec et al. (2004) found that the sla gene was more widely distributed in GAS than just serotype M3 strains. The sla gene was present in $10 \cdot 8 \%$ of 1189 GAS strains tested, including emm1, emm2, emm3, emm4, emm22, emm28 and emm7 (Nagiec et al., 2004). In our studies, we found the sla gene occurred at a lower rate $(4.65 \%)$ than those in other countries, and only four strains were sla positive, including emm18, emm101 and emm86, suggesting a horizontal transmission of the prophage-encoded gene in mainland China. Moreover, two of the four sla gene-positive strains could cause severe infection and death in mice (data not shown). Therefore, the sla gene is also an important virulence gene and plays a major role in invasive infection in $\mathrm{M}$ types other than M3.

The sil gene is the streptococcal invasion locus. Within this locus, a gene, silCR, is predicted to encode a 41 amino acid bacterial pheromone peptide that abrogates chemokine proteolysis. Hidalgo-Grass et al. (2004) showed that the M14 GAS invasive strains have a missense mutation (ATG $\rightarrow$ ATA) in the start codon of silCR, suggesting that the peptide might not be produced. We found that 11 strains carried the sil gene (Table 4), of which only one was from invasive infection. Sequence analysis of sil showed that these strains did not contain a missense mutation in the start codon of silCR, suggesting that the pheromone peptide might be produced in these strains and decrease host damage.

The overall rate of erythromycin resistance in our GAS isolates was high $(91 \cdot 8 \%)$ compared to reported rates of $2 \cdot 6 \%$ in the USA, $2 \cdot 1-4 \cdot 6 \%$ in Canada, $8 \cdot 6 \%$ in Finland and $32 \%$ in Hong Kong (Ho et al., 2003). We also found a high rate of resistance to tetracycline $(93.4 \%)$ compared to $4 \%$ in the USA and $53 \%$ in Hong Kong. The high rates of resistance that we found in mainland China are probably a reflection of the high level of antibiotic usage in China. Fortunately, the GAS strains in this study were still highly susceptible to penicillin, ceftriaxone, vancomycin, ciprofloxacin and chloramphenicol. Resistance to penicillin and ceftriaxone has not emerged in GAS, despite extensive use of $\beta$-lactam drugs in China. In general, the observations of GAS resistance to erythromycin, tetracycline and clindamycin are consistent with previous reports, despite a much higher drug-resistance rate (Ho et al., 2003; Bemer-Melchior \& Juvin, 2000; Deazavedo et al., 1999; Palavecino et al., 2001).

This epidemiological study has demonstrated different $\mathrm{emm}$ types and virulence factors in mainland China, and such data may help understand both pathogenesis and vaccine development. Fewer M3 and M14 types, lower carriage of the sla and sil genes, lack of the missense mutation in the start codon of silCR, and the continued susceptibility of GAS to antibiotics, may explain fewer severe clinical infections, such as NF and STSS, in mainland China.

\section{ACKNOWLEDGEMENTS}

We thank Dr Li Cheng for critical reading of and helpful suggestions for this manuscript. This work was supported by key programmes of the National Science and Technology Foundation of China (no. 2003BA712A04-06).

\section{REFERENCES}

Banks, D. J., Beres, S. B. \& Musser, J. M. (2002). The fundamental contribution of phages to GAS evolution, genome diversification and strain emergence. Trends Microbiol 10, 515-521.

Banks, D. J., Lei, B. \& Musser, J. M. (2003). Prophage induction and expression of prophage-encoded virulence factors in group A streptococcus serotype M3 strain MGAS315. Infect Immun 71, 7079-7086.

Bemer-Melchior, P. \& Juvin, M.-E. (2000). In vitro activity of the new ketolide telithromycin compared with those of macrolides against Streptococcus pyogenes: influences of resistance mechanisms and methodological factors. Antimicrob Agents Chemother 44, 2999-3002.

Beres, S. B. G. L., Sylva, K. D. \& Lei, B. B. (2002). Genome sequence of a serotype M3 strain of group A Streptococcus: phage-encoded toxins, the high-virulence phenotype and clone emergence. Proc Natl Acad Sci U S A 99, 10078-10083.

Bisno, A. L., Brito, M. O. \& Collins, C. M. (2003). Molecular basis of group A streptococcal virulence. Lancet Infect Dis 3, 191-200.

Broudy, T. B., Pancholi, V. \& Fischetti, V. A. (2001). Induction of lysogenic bacteriophage and phage-associated toxin from group A streptococci during coculture with human pharyngeal cells. Infect Immun 69, 1440-1443.

Carapetis, J., Robins-Browne, R., Martin, D., Shelby-James, T. \& Hogg, G. (1995). Increasing severity of invasive group A streptococcal disease in Australia: clinical and molecular epidemiological features and identification of a new virulent M-non-typeable clone. Clin Infect Dis 21, 1220-1227.

Chatellier, S., Ihendyane, N. \& Kansal, R. G. (2000). Genetic relatedness and superantigen expression in group A streptococcus serotype M1 isolates from patients with severe and non-severe invasive diseases. Infect Immun 68, 3523-3534.

Chiou, C.-S., Liao, T.-L., Wang, T.-H., Chang, H.-L., Liao, J.-C. \& Li, C.-C. (2004). Epidemiology and molecular characterization of Streptococcus pyogenes recovered from scarlet fever patients in Central Taiwan from 1996 to 1999. J Clin Microbiol 42, 3998-4006.

Collin, M. \& Olsen, A. (2001). Effect of speB and EndoS from Streptococcus pyogenes on human immunoglobulins. Infect Immun 69, 7187-7189.

Cunningham, M. W. (2000). Pathogenesis of group A streptococcal infections. Clin Microbiol Rev 13, 470-511.

Deazavedo, J. C. S., Yeung, R. H., Bast, D. J., Duncan, C. L., Borgia, S. B. \& Low, D. E. (1999). Prevalence and mechanisms of macrolide resistance in clinical isolates of group A streptococci from Ontario, Canada. Antimicrob Agents Chemother 43, 2144-2147.

Goshorn, S. C. \& Schlievert, P. M. (1989). Bacteriophage association of streptococcal pyrogenic exotoxin type C. J Bacteriol 171, 3068-3073.

Hidalgo-Grass, C., Ravins, M., Dan-Goor, M., Jaffe, J. \& Moses, A. E. (2002). A locus of group A streptococcus involved in invasive disease and DNA transfer. Mol Microbiol 46, 87-99.

Hidalgo-Grass, C., Dan-Goor, M. \& Maly, A. (2004). Effect of a bacterial pheromone peptide on host chemokine degradation in 
group A streptococcal necrotizing soft-tissue infections. Lancet 363 , 696-703.

Ho, P. L., Johnson, D. R., Yue, A. W. Y., Tsang, D. N. C., Que, T. L., Beall, B. \& Kaplan, E. L. (2003). Epidemiologic analysis of invasive and noninvasive group A streptococcal isolates in Hong Kong. J Clin Microbiol 41, 937-942.

Holm, S. E., Norrby, A., Bergholm, A. M. \& Norgren, M. (1992). Aspects of pathogenesis of serious group A streptococcal infections in Sweden, 1988-1989. J Infect Dis 166, 31-37.

Ikebe, T. \& Wada, A. (2002). Dissemination of the phage-associated novel superantigen gene sepL in recent invasive and noninvasive Streptococcus pyogenes M3/T3 isolates in Japan. Infect Immun 70, 3227-3233.

Kaul, R. A., McGeer, A., Low, D. E., Green, K., Schwartz, B. \& Simor, A. E. (1997). Population-based surveillance for group A Streptococcal necrotizing fasciitis, clinical features, prognostic indicators, and microbiologic analysis of seventy-seven cases. Am J Med 103, $18-24$.

Kiska, D. L., Thiede, B., Caracciolo, J. \& 7 other authors (1997). Invasive group A streptococcal infections in North Carolina: epidemiology, clinical features, and genetic and serotype analysis of causative organisms. J Infect Dis 176, 992-1000.

Kotb, M. (1995). Bacterial pyrogenic exotoxins as superantigens. Clin Microbiol Rev 8, 411-426.

Louie, L. \& Simor, A. E. (1998). Diagnosis of group A streptococcal necrotizing fasciitis by using PCR to amplify the streptococcal pyrogenic exotoxin B gene. J Clin Microbiol 36, 1769-1771.

Musser, J. M., Kupur, V. \& Szeto, J. (1995). Genetic diversity and relationships among Streptococcus pyogenes strains expressing serotype M1 protein: recent international spread of a subclone causing episodes of invasive disease. Infect Immun 63, 994-1003.

Nagiec, M. J., Lei, B., Parker, S. K. \& Vasil, M. L. (2004). Analysis of a novel prophage-encoded group A streptococcus extracellular phospholipase A2. J Biol Chem 279, 45909-45918.

O'Brien, K. L., Beall, B., Barrett, N. L. \& 8 other authors (2002). Epidemiology of invasive group A streptococcus disease in the United States, 1995-1999. Clin Infect Dis 35, 268-276.

Palavecino, E. L., Riedel, I., Berrios, X., Bajaksouzian, S., Johnson, D., Kaplan, E. \& Jacobs, M. J. (2001). Prevalence and mechanisms of macrolide resistance in Streptococcus pyogenes in Santiago, Chile. Antimicrob Agents Chemother 45, 339-341.

Schmitz, F.-J., Beyer, A. \& Charpentier, E. (2003). Toxin-gene profile heterogeneity among endemic invasive European group A streptococcal isolates. J Infect Dis 188, 1578-1586.

Vlaminckx, B. J. M., Mascini, E. M., Schellekens, J., Schouls, L. M., Pauw, A., Fluit, A. C., Novak, R., Verhoef, J. \& Schmitz, F. J. (2003). Site-specific manifestations of invasive group A streptococcal disease: type distribution and corresponding patterns of virulence determinants. J Clin Microbiol 41, 4941-4949.

Watanabe, Y., Todome, Y., Ohkuni, H., Sakurada, S., Ishikawa, T., Yutsudo, T., Fischetti, V. A. \& Zabriskie, J. B. (2002). Cysteine protease activity and histamine release from the human mast cell line HMC-1 stimulated by recombinant streptococcal pyrogenic exotoxin B/streptococcal cysteine protease. Infect Immun 70, 3944-3947.

Yu, C. E. \& Ferretti, J. J. (1991). Molecular characterization of new group A streptococcal bacteriophages containing the gene for streptococcal erythrogenic toxin A (sepA). Mol Gen Genet 231, $161-168$. 